\section{WEB WATCH}

Passionate about peroxisomes

Peroxisomes are spherical organelles that compartmentalize many metabolic reactions. If this introduction doesn't sound overly appealing, go to the peroxisome website created and maintained in Stephen J. Gould's laboratory - and you'll develop a deep love for these organelles.

The site is unique in that the information is pitched at three levels: scientist, physician or layperson. The scientist will find a

comprehensive and up-todate review of peroxisome biology, whereas the physician's version focuses on peroxisomal disorders. And your hypochondriac grandfather will be able to surf the layperson's version, and discover all sorts of peroxisome-related diseases that he might have.

In the scientist's version, after the first page of introduction, you can go in two directions: peroxisome biogenesis or biochemistry of the peroxisome. The two directions then zoom in on individual molecule pages or enzymatic reaction pages, respectively. The molecule pages are short summaries about the different peroxins, with a comprehensive bibliography. There are also links to sidetrack pages that comment on controversies in the field. The only possible concern about the site is that as it is the creation of a single laboratory, these comments might carry some bias.

The site has no fancy web stuff - the cartoons are simple, and there are no animations or flashing headers - but the graphics, although simple, are pleasant to look at. More importantly, the information is beautifully organized. Peroxisomes are far less well understood than other organelles, so the amount of information is not yet overwhelming. But the creators of this site have demonstrated here what you can do with a bit of time and love for your research.

Raluca Gagescu

\title{
Matchmaking for chromosomes
}

The oscillation of an organism between haploid and diploid stages is the very essence of sexual reproduction. Thus the ability to accurately pair and separate homologous chromosomes during meiosis is essential for any sexually reproducing organism. Reporting in Nature, Graham Moore and colleagues at the John Innes Centre, Norwich, UK, have explored how this fidelity is maintained.

Pairing of homologous chromosomes is difficult enough in a diploid organism, but many plants are polyploid with, in effect, several genomes sharing each cell. Pairing of chromosomes occurs mainly through the centromeres, so in wheat, a hexaploid, each centromere has five very similar potential partners, only one of which is its true homologue. One might expect that this complexity would result in meiosis taking longer in polyploid species compared with diploids - but in fact it is faster. This is probably because, while homologous chromosomes come

\section{TRANSLOCATION}

\section{Paradigm lost}

The accepted model for protein import into organelles is that targeting is mediated by receptors that recognize signal sequences. Once cargo proteins have reached the correct membrane, they unfold, thread through the translocon and refold in the lumen of the organelle. Of course, nothing is ever simple, and Dammai and Subramani now report in Cell that Pex5 - the receptor for the peroxisomal targeting signal 1 - actually cycles in and out of peroxisomes.

The authors constructed together only at the onset of meiotic prophase in diploids, polyploids pair their chromosomes long before meiosis begins.

In 1958, the agricultural geneticist Sir Ralph Riley identified the $P H 1$ locus in wheat to be essential for correct homologous chromosome pairing and recombination. As a result of his work, a single mutant carrying a deletion of this locus has been used in wheat breeding ever since. Its activity has remained controversial, however, and even its composition is unclear.

Moore and colleagues used confocal microscopy to investigate $\mathrm{PH} 1$ function after the pairing of chromosomes in developing xylem cells from wheat. They were particularly concerned with a stage at the beginning of meiosis when a 'telomere bouquet' forms. Here, all of the telomeres (red in the figure) come together at one side of the dividing cell, whereas the centromeres (green) congregate at the other. It was difficult to distinguish paired from single centromeres,

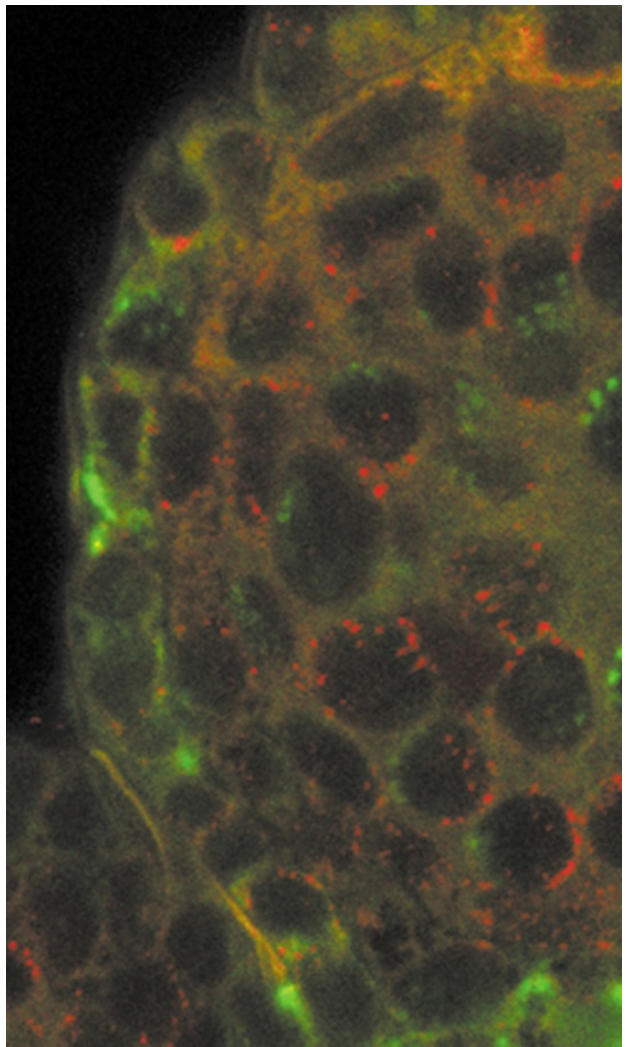

but in the absence of the $\mathrm{PH} 1$ locus there were more centromere pairs and fewer singlets than in its presence. In wheat/rye hybrid plants, the chromosomes from the different parents could be identified, allowing the authors to deduce that there was less mispairing of centromeres in the presence of $\mathrm{PH} 1$.

The molecular mechanism by which the PH1 locus weeds out incorrect centromere pairings before meiosis begins - so that only correct pairings are carried into cell division chimaeras consisting of a PTS2 signal sequence, a prethiolaseprocessing site, a FLAG epitope and Pex5. The FLAG epitope can be detected by two antibodies: M2, which recognizes it in any context; and M1, which recognizes it only when it is amino terminal — as, for example, after cleavage of the construct by the prethiolase protease. Because the protease cleaves prethiolase only in the peroxisome matrix, cleavage of the construct is diagnostic of entry into peroxisomes.

After expression in HeLa cells, the chimaera was cleaved, indicating that Pex 5 had entered peroxisomes. Pex5 was also exported from peroxisomes, as the processed form was largely cytosolic. Peroxisomeassociated, processed Pex5 was only partly protected from protease digestion, indicating that it was no longer inside the peroxisome but had docked again to the organelle membrane. Last, kinetic data indicated that processed Pex5 could re-enter peroxisomes, as the appearance of the peroxisomal, processed form accounted for the disappearance of the processed and unprocessed forms from the cytosol.

So peroxisomal import seems to be diametrically opposed to the principles derived from import into mitochondria. Although it remains to be shown that cycling of Pex5 between the cytosol and the peroxisome is relevant for cargo transport, this shuttling mechanism is reminiscent of nuclear transport. The directionality of nuclear 